\title{
Anxiety modulates the degree of attentive resources required to process emotional faces
}

\author{
ELAINE FOX, RICCARDO RUSSO, and GEORGE A. GEORGIOU \\ University of Essex, Colchester, England
}

\begin{abstract}
The present study contributes to the ongoing debate over the extent to which attentive resources are required for emotion perception. Although fearful facial expressions are strong competitors for attention, we predict that the magnitude of this effect may be modulated by anxiety. To test this hypothesis, healthy volunteers who varied in their self-reported levels of trait and state anxiety underwent an attentional blink task. Both fearful and happy facial expressions were subject to a strong attentional blink effect for low-anxious individuals. For those reporting high anxiety, a blink occurred for both fearful and happy facial expressions, but the magnitude of the attentional blink was significantly reduced for the fearful expressions. This supports the proposals that emotion perception is not fully automatic and that anxiety is related to a reduced ability to inhibit the processing of threat-related stimuli. Thus, individual differences in self-reported anxiety are an important determinant of the attentional control of emotional processing.
\end{abstract}

Attentional systems are finely tuned to enable the efficient processing of task-relevant stimuli. However, the extent to which unattended stimuli are processed remains a fundamental question. Several classes of evidence suggest that emotional stimuli may represent a special category that requires few attentional resources in order to be fully processed. For example, patients with clinical neglect due to damage to the right parietal lobe demonstrate less spatial extinction for emotional facial expressions than for neutral expressions (Fox, 2002; Vuilleumier \& Schwartz, 2001a, 2001b). Likewise, photographs of angry facial expressions are detected far more efficiently in visual search tasks than are those of happy faces (Eastwood, Smilek, \& Merikle, 2001; Fox et al., 2000; Öhman, Lundqvist, \& Esteves, 2001), and spiders and snakes are detected more easily than flowers or mushrooms (Öhman, Flykt, \& Esteves, 2001). In conditioning experiments, larger skin conductance responses and greater resistance to extinction have been found for aversively conditioned angry facial expressions than for happy or neutral facial expressions (Esteves, Parra, Dimberg, \& Öhman, 1994). Neuroimaging studies have shown that emotional stimuli, especially fearful facial expressions, elicit strong activation of the amygdala (Morris et al., 1996) even when the expressions are spatially unattended (Vuilleumier, Armony, Driver, \& Dolan, 2001) or backward masked and inaccessible to

This study was conducted at the University of Essex Psychology Department and supported by Project Grant 064290/Z/01/Z awarded to E.F. and R.R. by the Wellcome Trust. We thank Sophie Lovejoy for help with programming this experiment, and we are grateful for the helpful comments of the reviewers of an earlier version of this article. Correspondence should be addressed to E. Fox, Department of Psychology, University of Essex, Wivenhoe Park, Colchester CO4 3SQ, England (e-mail: efox@essex.ac.uk). conscious awareness (Morris, Öhman, \& Dolan, 1999; Whalen et al., 1998). This functional imaging work is particularly interesting in that the enhanced activation of the amygdala in response to threat-related stimuli has been shown to modulate modality-specific sensory cortical areas (Armony \& Dolan, 2001; Morris et al., 1998). The implications of these findings are that the amygdala is activated directly by the presence of threat-related stimuli and that cortical areas are then modulated via reentrant projections from the amygdala to the cortex (Amaral \& Price, 1984). This mechanism would serve to prioritize the processing of emotionally significant stimuli (Anderson \& Phelps, 2001; Vuilleumier, Richardson, Armony, Driver, \& Dolan, 2004).

\section{Emotion Processing and Attention}

Recently, there has been controversy in the neuroimaging literature regarding the extent to which the amygdala's response to emotional stimuli is dependent on attention. On the one hand, enhanced amygdala activity in response to unattended or backward-masked threat-related stimuli has been observed (Armony \& Dolan, 2001; Morris et al., 1999; Vuilleumier et al., 2001; Whalen et al., 1998) and a subcortical pathway allowing for the preattentive analysis of environmental threat has been proposed (Morris et al., 1999). This suggests that few attentional resources are required for emotion processing, and behavioral tasks using an attentional blink (AB) paradigm also support this notion. The $\mathrm{AB}$ paradigm requires people to identify two successive targets embedded in a rapidly presented stream of items - usually letters or words. The AB effect is revealed by an impaired ability to identify the second target (T2) when a response is required to the first target (T1) in comparison with when no response is required to $\mathrm{T} 1$. Typically, identification of T2 is impaired when it is 
presented within about $500 \mathrm{msec}$ following $\mathrm{T} 1$ - hence the analogy to a blink effect. The AB is assumed to reflect attentional capacity limitations when two targets are presented in rapid succession (Raymond, Shapiro, \& Arnell, 1992; Shapiro, Arnell, \& Raymond, 1997). Whereas AB effects are common for words and other people's names, when one's own name is presented as T2 a blink does not occur (Shapiro, Caldwell, \& Sorensen, 1997), indicating that emotionally salient stimuli may be immune to the AB. In support of this, in healthy individuals T2 words with very strong negative affect produced no $\mathrm{AB}$ effect and were identified more frequently than neutral words, whereas patients with amygdala damage showed equivalent AB effects for negative and neutral words (Anderson \& Phelps, 2001). In an extension of this work, emotionally arousing verbs (both pleasant and unpleasant) have been found to attenuate the $\mathrm{AB}$ effect more than do neutral verbs, whereas pleasant and unpleasant verbs rated as low in terms of emotional arousal did not attenuate the AB effect (Keil \& Ihssen, 2004). Thus, emotionally arousing stimuli seem to be less subject to top-down attentional control.

On the other hand, some studies indicate that attentional resources may be required for the analysis of emotional stimuli (Pessoa, Kastner, \& Ungerleider, 2002; Pessoa, McKenna, Gutierrez, \& Ungerleider, 2002; Phillips et al., 2004). Research showing amygdala activation to unattended threat stimuli, for example, may have been inadvertently flawed in that the primary task did not require sufficient attentional resources to ensure that the threat stimuli were genuinely unattended (Pessoa, Kastner, \& Ungerleider, 2002; Pessoa, McKenna, et al., 2002). Evidence in support of this was shown in that, when a high perceptual load task was presented as the primary task, unattended fearful faces no longer elicited activation of the amygdala (Pessoa, McKenna, et al., 2002). This reflects behavioral results showing that when perceptual resources are fully occupied, unattended stimuli are not processed beyond a fairly superficial level (Lavie, 1995; Lavie \& Fox, 2000). However, recent evidence obtained using event-related brain potentials (ERPs) has indicated that attention may be necessary for emotional processing even when perceptual load is low (Holmes, Vuilleumier, \& Eimer, 2003). When faces were focally attended, greater frontal positivity in response to fearful faces was observed starting about $100 \mathrm{msec}$ after stimulus onset. However, when the fearful faces were unattended, this effect was completely eliminated, suggesting a strong attentional gating of emotional processing even under conditions of low perceptual load (Holmes et al., 2003).

\section{The Role of Anxiety}

An explanation of these apparently contradictory results may be gained from consideration of research in the clinical cognition literature. To illustrate, several studies show that people who report high levels of trait or state anxiety are more distracted than others by irrelevant emotional words in Stroop color-naming tasks (Williams, Mathews, \& MacLeod, 1996), are faster to detect probes when they occur in the location of fearful or angry faces as opposed to neutral faces (Fox, 2002; Mogg \& Bradley, 1999), take longer to disengage their attention from angry faces or threat-related pictures (Fox, Russo, Bowles, \& Dutton, 2001; Georgiou et al., 2005; Yiend \& Mathews, 2001), and are quicker to follow the gaze of fearful faces relative to that of neutral faces (Mathews, Fox, Yiend, \& Calder, 2003). These patterns of differential attentional biases to emotional stimuli, however, do not occur for those reporting low levels of anxiety. Behavioral evidence also demonstrates that high anxiety is associated with an impaired ability to actively inhibit threat-related emotional material (Derryberry \& Reed, 2002; Fox, 1994), and anxiety is associated with a failure to activate the rostral anterior cingulate cortex (ACC), further indicating a reduced ability to recruit attentional control (Bishop, Duncan, Brett, \& Lawrence, 2004). Thus, it can be hypothesized that the processing of emotional faces is generally under top-down attentional control (at least when attention is occupied by a relatively difficult task) but that emotion processing may proceed more automatically for those with high levels of anxiety. The apparent inconsistency in the neuroimaging studies in the role of attention in emotion processing may therefore be due to unequal mixes of anxious and nonanxious people in the samples. The suggestion here is that variation in anxiety may be a key determinant of whether or not attention successfully gates emotion processing.

There are two previous reports of the influence of selfreported anxiety on $\mathrm{AB}$ effects, neither of which is directly relevant to the present hypothesis. Arend and Botella (2002) manipulated the valence of T1 words (neutral vs. threat related) and found that the requirement to process a threat-related word at $\mathrm{T} 1$ resulted in an attenuated $\mathrm{AB}$ for neutral $\mathrm{T} 2$ words for those reporting high anxiety but not for those reporting low anxiety. Arend and Botella concluded that the processing of threat is automatic for those with high anxiety, which means that less attention is required, resulting in a reduced blink effect. This finding is relevant for our study but does not directly shed light on the extent to which attention gates emotion processing. To answer this question, we need to manipulate the valence of $\mathrm{T} 2$ rather than that of $\mathrm{T} 1$. In a novel version of the $\mathrm{AB}$ task, Barnard, Ramponi, Battye, and Mackintosh (2005) also examined the AB task in relation to anxiety. Groups of participants reporting high and low levels of anxiety were required to report target words of a particular semantic category, and the relations between each target and its preceding distractor were manipulated. A complex relationship among the semantic associations between targets and distractors, the onset of blink effects, and the level of self-reported anxiety was found. Since separate responses to T1 and T2 were not required in this study, our primary hypothesis that anxiety may be a key determinant of the degree to which attention successfully gates emotion processing was not directly addressed. For a direct examination of the extent to which attention is required for emotion perception and whether anxiety modulates this effect, the ideal task is an AB task using facial expressions in which the valence of T2 targets is manipulated. 


\section{The Present Study}

We tested this hypothesis by presenting an $\mathrm{AB}$ task to two groups of participants who were selected on the basis of their scores on a standardized scale of trait anxiety (Spielberger, 1983). We note, however, that the two groups also differed in level of state anxiety, so the labels high anxious (HA) and low anxious (LA) are used. This experiment is the first test of whether $\mathrm{AB}$ effects might occur for emotional faces. We specifically chose faces as stimuli because they have been used as stimuli in recent brain imaging studies on attention and emotion (e.g., Pessoa, McKenna, et al., 2002; Vuilleumier et al., 2001). These neuroimaging studies have reignited the historic early-late selection debate regarding the extent to which attentional resources are necessary for semantic processing of stimuli (see, e.g., Driver \& Tipper, 1989; Lavie \& Tsal, 1994). We considered that if processing of fear-relevant facial expressions occurs automatically, then the magnitude of the $\mathrm{AB}$ effect should be attenuated for these faces relative to emotional faces with happy expressions.

We presented rapid serial visual presentation (RSVP) streams of distractors, and the first target to be responded to (T1) was a picture of either mushrooms or flowers, which had to be categorized at the end of the RSVP stream. The T2 was either a fearful or a happy facial expression that was embedded in a stream of faces with neutral expressions and that had to be categorized at the end of each RSVP. On each trial, 15 photographs were rapidly presented in series, for $110 \mathrm{msec}$ each. On dual-task trials, the requirement was to detect whether a fearful or a happy facial expression (T2) had been presented and then to categorize nonfacial stimuli (T1) as either mushrooms or flowers. On single-task trials, T1s were simply to be ignored and a response was required only for T2. On dual-task trials, the response to T2 was required prior to that to $\mathrm{T} 1$ in order to equate the temporal interval for responses to $\mathrm{T} 2$ targets on both single- and dual-task trials. All other background distractor stimuli were neutral facial expressions. (Figure 1 illustrates a typical trial in the experiment.) If the processing of emotional faces is under top-down attentional control for LA people but not for HA people, then a statistical interaction should be observed between anxiety status and the appearance of an $\mathrm{AB}$ for emotional faces. More specifically, the detection of fearful facial expressions should be subject to a stronger $\mathrm{AB}$ for LA individuals than for HA individuals.

\section{METHOD}

\section{Participants}

Fourteen people (10 female) scoring above 45 on the State-Trait Anxiety Inventory questionnaire (Spielberger, 1983; range, 20-80) and 14 (10 female) scoring below 35 on this questionnaire were

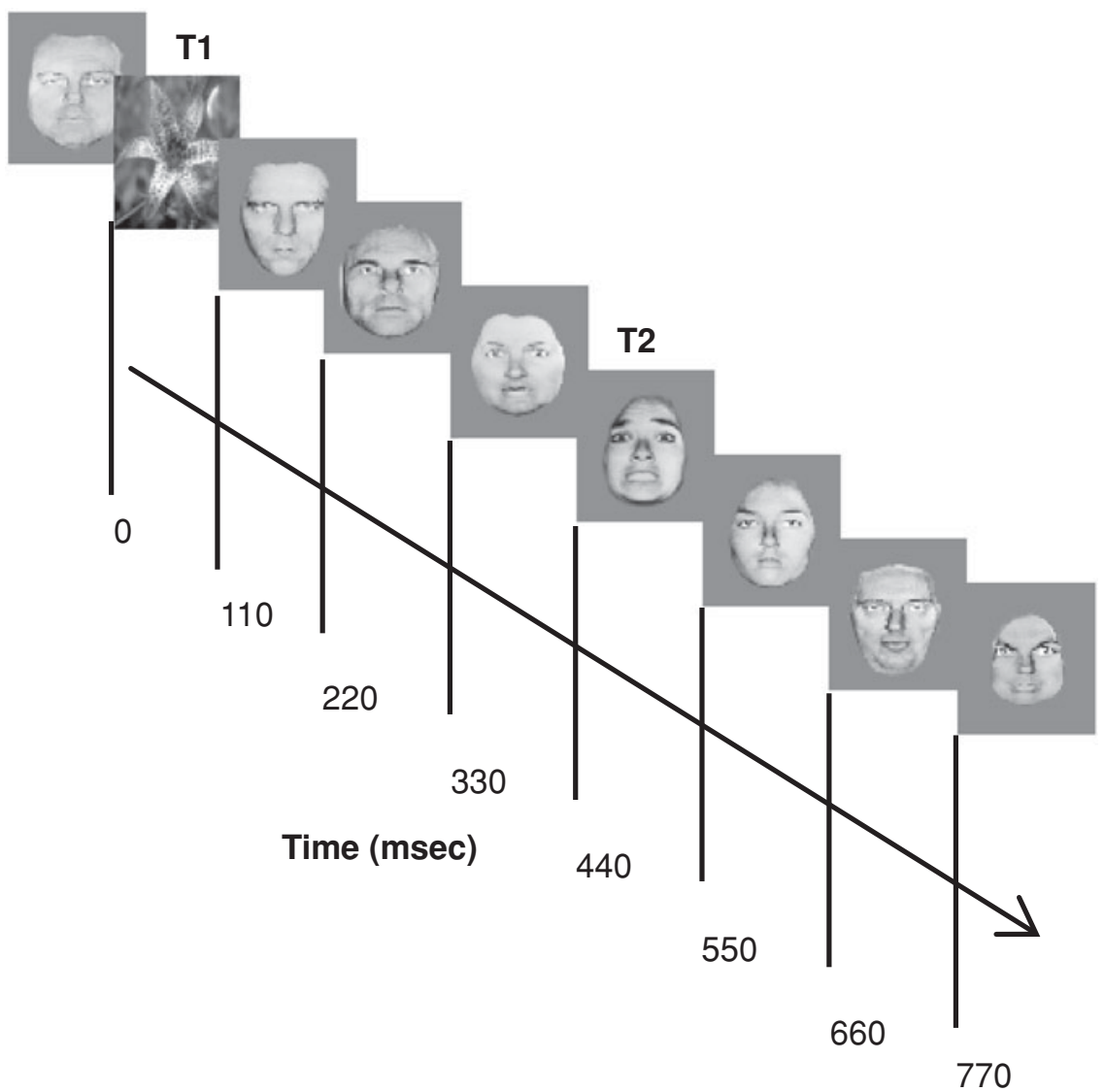

Figure 1. An example of a typical trial in the experiment. This example shows a fearful face at $\mathrm{T} 2$. 
recruited to participate in the study. All of the participants were between 18 and 32 years of age, and each participant was either paid $£ 5$ or awarded course credit for $45 \mathrm{~min}$ of his or her time. The study was approved by the University of Essex Ethics Committee.

\section{Stimuli}

The stimuli were black and white photographs of fearful, happy, and neutral faces selected from a standard set of pictures representing facial affect (Ekman \& Friesen, 1975), as well as other photographs used in our laboratory. An independent sample of 20 students from the same population as the participants rated these faces on a set of seven-point scales for threat relevance $(1=$ not at all relevant, $7=$ extremely relevant $)$, emotionality $(1=$ not at all emotional, $7=$ extremely emotional $)$, and arousal $(1=$ not at all arousing, $7=e x-$ tremely arousing). The fearful expressions were rated as more threat relevant $(M=5.9)$ than either the happy (1.1) or the neutral distractor (1.5) expressions (all $p \mathrm{~s}<.001)$. However, the fearful and happy expressions did not differ from each other in terms of either emotionality (5.1 and 5.2, respectively) or arousal (4.3 and 4.0, respectively), whereas both affective categories were rated as being more emotional and arousing than the neutral expressions $(2.2, p \mathrm{~s}<.001)$. Black and white photographs of mushrooms and flowers (which were matched for brightness and complexity using Adobe Photoshop) were also selected from previous research in our laboratory, and these had been rated as emotionally neutral in previous studies. Stimuli were presented at a viewing distance of about $60 \mathrm{~cm}$ at the center of a computer screen against a gray background. Stimulus presentation and data collection were controlled by a G4 PowerMac computer

\section{Procedure}

Each trial consisted of 15 stimuli, each presented for $110 \mathrm{msec}$ without any interstimulus interval. Each sequence contained one picture of flowers or mushrooms (T1, at serial position $2,3,4$, or 5 in the 15 -item sequence). The distractor stimuli were pictures of neutral faces, and the target stimuli (T2) were pictures of emotional (fearful or happy) faces. On T2-present trials, emotional faces appeared equally often in positions $2,3,4,5,6$, and 7 following $\mathrm{T} 1$ (i.e., serial positions $4-13$ in the 15 -item sequence). This design corresponded to T1-T2 intervals of 220, 330, 440, 550, 660, and $770 \mathrm{msec}$ (see Figure 1 for a sample trial). Half of the trials were single-task trials, in which the participants were instructed to ignore the flowers or the mushrooms and to report at the end of each trial (by pressing one of two keys) whether or not an emotional face had been presented in the stream. Thirty-eight of these trials occurred for each T1-T2 interval. In dual-task trials, the participants were required to indicate whether or not an emotional face had been presented by pressing one of two keys and then to indicate whether the nonface stimulus (T1) was either flowers or mushrooms by again pressing one of two keys. The participants gave these responses at the end of each trial, and the $\mathrm{T} 2$ judgment was always given before the $\mathrm{T} 1$ judgment on dual-task trials. As in the single-task condition, 38 of these trials occurred for each T1-T2 interval (220-770 msec).

The experiment consisted of a total of 456 trials divided into 228 single-task trials and 228 dual-task trials (each group presented in four blocks of 57 trials). In both tasks, T2 was present on $63 \%$ of the trials (i.e., on 24 trials in each T1-T2 interval); half (12) of the T2s depicted fearful expressions, and half depicted happy expressions. There were 48 individual T2 photographs ( 24 fearful expressions and 24 happy expressions), and each of these faces was presented six times during the experiment (three times in the single task and three times in the dual task). Individual expressions were repeated equally often across the various conditions. On 14 trials $(37 \%)$ in each T1-T2 interval, T2 was absent. In order to maximize the number of T2-present trials without making the experiment overly long, the probability of T2's being present and the probability of its being absent were not equal. This aspect of the design also gave some face validity to the experiment, since the attentional blink itself often leads to the impression that T2 trials are very infrequent. Half of each anxiety group $(n=7)$ received the four blocks of single-task trials followed by four blocks of dual-task trials, whereas the other half received the blocks in the reverse order (i.e., dual task followed by single task).

\section{RESULTS}

\section{Demographic Data and Responses to T1}

The HA group reported higher levels of both trait and state anxiety than did the LA group (both $p s<.001$ ). As is shown in Table 1, both groups were very accurate in the $\mathrm{T} 1$ task (mean accuracy $=90 \%$ ) and accuracy did not differ between trials that subsequently contained a fearful emotional expression and those that subsequently contained a happy one.

\section{Responses to $\mathrm{T} 2$}

The false alarm rate on T2-absent trials was higher in the dual-task relative to the single-task condition for the HA group $[t(14)=4.2, p<.001$, two-tailed], with a trend in the same direction for the LA group $[t(14)=1.2, p<$ .07 , two-tailed; see Table 1].

The main analysis was conducted on the percentage of fearful and happy faces correctly identified on T2-present trials. Only dual-task trials in which the response to T1 had been correct were included in the analysis. Figure 2 shows the percentage correct in the single- and dual-task conditions as a function of anxiety status (high vs. low), valence of emotional face (fearful vs. happy), and T1-T2 lag $(220,330,440,550,660$, and $770 \mathrm{msec})$. As can be seen, an AB was apparent for the LA group for both fearful and happy expressions at the shorter lags (220, 330, and $440 \mathrm{msec}$ ) but not at the longer lags $(550,660$, and $770 \mathrm{msec}$ ). Although an AB was also apparent for the HA group at shorter lags for happy expressions, it seemed to be attenuated for fearful faces. This impression was substantiated by a significant interaction between anxiety status, task (single vs. dual), valence of T2, and lag $[F(5,130)=3.4, p<.01]$.

Further analysis revealed that the single-task performance did not differ between HA and LA groups or between fearful and happy expressions and remained fairly constant across all lags $(M=91.5 \%)$. Therefore, for ease of exposition the subsequent analyses focus on responses

Table 1

Mean Scores (and Standard Deviations) for Questionnaire Measures of Trait and State Anxiety, Performance on the T1 Task, and False Alarms on the

T2-Absent Trials for High Trait Anxious (HA; $n=14$ ) and Low Trait Anxious (LA; $n=14)$ Groups

\begin{tabular}{|c|c|c|c|c|}
\hline \multirow[b]{2}{*}{ Measure } & \multicolumn{2}{|c|}{ HA } & \multicolumn{2}{|c|}{ LA } \\
\hline & $M$ & $\overline{S D}$ & $M$ & $S D$ \\
\hline Trait anxiety & 53.3 & 6.3 & 25.9 & $4.7^{* *}$ \\
\hline State anxiety & 50.6 & 8.7 & 26.9 & $6.1^{*}$ \\
\hline$\%$ correct on $\mathrm{T} 1$ (fearful $\mathrm{T} 2$ ) & 89.2 & 2.9 & 90.4 & 3.4 \\
\hline$\%$ correct on T1 (happy T2) & 89.4 & 3.1 & 90.3 & 3.1 \\
\hline False alarms on $\mathrm{T} 2$ (single task) & 7.4 & 1.8 & 6.1 & 2.3 \\
\hline False alarms on T2 (dual task) & 8.3 & 2.3 & 6.6 & 2.5 \\
\hline
\end{tabular}

${ }^{* *} p<.001$. 

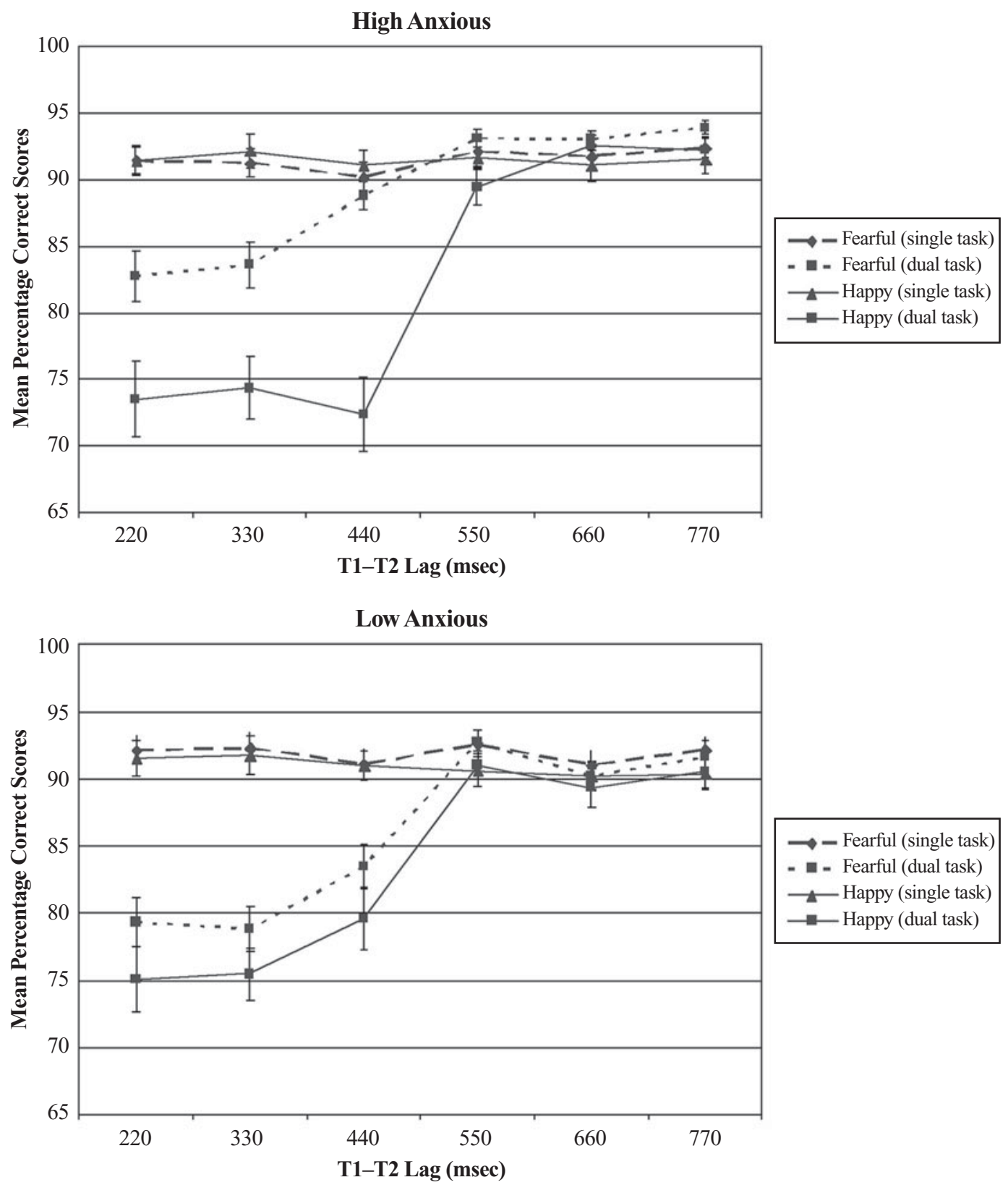

Figure 2. Mean percentage correct scores as a function of anxiety status (high vs. low), valence of T2 (fearful vs. happy), type of task (single vs. dual), and SOA (220-770 msec).

to T2 under dual-task conditions, and a series of planned $t$ tests compared the AB effects for fearful and happy expressions, respectively, at each of the lags. Given six comparisons for each anxiety group, the Bonferroni correction implies that a one-tailed test is significant at $p<.008$.

\section{Responses to T2 on Dual-Task Trials}

There was a significant interaction between anxiety status, valence of $\mathrm{T} 2$, and lag $[F(5,130)=3.1, p<.01]$ on dual-task performance. Further analysis of the HA group showed significant main effects for valence of $\mathrm{T} 2[F(1,13)=17.1, p<.001]$ and $\operatorname{lag}[F(5,65)=58.0$, $p<.001]$, and these were qualified by a significant valence $\times$ lag interaction $[F(5,65)=8.4, p<.001]$. For the LA group, a valence-of-T2 $\times$ lag ANOVA showed a main effect only for lag $[F(5,65)=30.8, p<.001]$, indicating that accuracy improved as lag length increased. In contrast to the results for the HA group, the valence of T2 did not affect performance.

\section{Planned Contrasts}

To establish the presence of $\mathrm{AB}$ effects, performance in the single task and that in the dual task were compared for each lag with paired $t$ tests. For the HA group, the detec- 
tion of happy faces was impaired in the dual task relative to the single task for lags of $220[t(13)=6.3, p<.001]$, $330[t(13)=7.1, p<.001]$, and $440[t(13)=6.2, p<$ $.001] \mathrm{msec}$, whereas the detection of fearful faces was impaired in the dual-task condition only for lags of 220 $[t(13)=4.3, p<.001]$ and $330[t(13)=4.2, p<.001]$ msec. For the LA group, an AB was observed for happy faces at lags of $220[t(13)=7.1, p<.001], 330[t(13)=$ $7.3, p<.001]$, and $440[t(13)=5.1, p<.001] \mathrm{msec}$, and for fearful faces at lags of $220[t(13)=6.4, p<.001]$, $330[t(13)=6.1, p<.001]$, and $440[t(13)=3.7, p<$ $.01]$ msec. An attentional blink index was computed by calculating the difference in accuracy between single- and dual-task conditions for each valence (fearful and happy) at each lag, and this is shown in Figure 3. As can be seen in Figure 3, for the HA group the AB was larger for happy faces relative to fearful faces for the 220 -msec [17.9\% vs. $8.6 \% ; t(13)=2.5, p<.014], 330-\mathrm{msec}[17.8 \%$ vs. $7.7 \%$; $t(13)=3.4, p<.002]$, and $440-\mathrm{msec}[18.7 \%$ vs. $1.3 \%$; $t(13)=5.3, p<.001]$ lags. In contrast, for the LA group there was no difference in the magnitude of the AB effects for fearful and happy faces at any lag.

\section{Differences Between Anxiety Groups in the Magnitude of the Attentional Blink Effect}

The magnitude of the AB effect for happy expressions did not differ between HA and LA groups for lags of 220, 330 , and $440 \mathrm{msec}$. However, for fearful faces, the AB at a lag of $220 \mathrm{msec}$ tended to be lower for the HA group than for the LA group [ $8.6 \%$ vs. $12.8 \% ; t(26)=1.5, p<.08$ ], as it was for lags of $330 \mathrm{msec}[7.7 \%$ vs. $13.5 \% ; t(26)=$ $2.0, p<.03]$ and $440 \mathrm{msec}[1.3 \%$ vs. $7.6 \% ; t(26)=2.8$, $p<.01]$. A correlational analysis showed that the magnitude of the attentional blink for fearful faces was negatively correlated with both trait anxiety $(r=-.49, p<$ $.009)$ and state anxiety $(r=-.50, p<.006)$ at a lag of $440 \mathrm{msec}$, so that higher levels of anxiety were associated with lower levels of attentional blink. This correlation survived correction for multiple comparisons. However, the correlations for lags of 220 and $330 \mathrm{msec}$ failed to reach statistical significance, and no correlations were observed between anxiety measures and the magnitude of blink effects for happy faces.

\section{DISCUSSION}

Two important results emerge from the present experiment. First, an AB effect occurred for fearful faces, even for highly anxious people. This provides behavioral evidence that the processing of fearful faces is not completely automatic. The question of automaticity of emotion processing is controversial, with some claiming that emotion, especially fear, is processed with few attentional resources (e.g., Vuilleumier et al., 2001) and others arguing that attention is always required for emotion perception (e.g., Pessoa, McKenna, et al., 2002). The present result implies that the processing of fearful expressions is not fully automatic, since $\mathrm{AB}$ effects did occur for these stimuli.
Second, the results show that anxiety modulates the extent to which attention is required for the processing of fearful faces. For LA individuals, both fearful and happy expressions were subject to a strong $\mathrm{AB}$, which suggests that attentional resources are indeed required for the processing of emotional expressions in these individuals. The HA individuals showed a different pattern in that, whereas an $\mathrm{AB}$ was observed for both fearful and happy expressions, the magnitude of the blink was significantly attenuated for fearful expressions and was also more short-lived (up to $330 \mathrm{msec}$ ) than that observed for happy faces (up to $440 \mathrm{msec}$ ). This supports the findings of the clinical cognition literature that emotion processing is less affected by attentional control in people with high levels of trait anxiety (Derryberry \& Reed, 2002; Fox, 1994, 2002; Mogg \& Bradley, 1999).

The role of anxiety in modulating attentional processes in the perception of emotional stimuli may help explain the inconsistency in recent neuroimaging studies, some of which have shown that emotion processing can proceed without attention (e.g., Armony \& Dolan, 2001; Vuilleumier et al., 2001) whereas others report strong effects of attention on emotion processing (Holmes et al., 2003; Pessoa, McKenna, et al., 2002). The present results suggest that emotion may proceed fairly automatically for those reporting high levels of anxiety, so that fearful expressions may "break through" the AB to a greater extent. However, for those with low levels of anxiety, all items are subjected to a strong AB up to around 500 msec regardless of their emotional significance. Converging evidence for this proposal has been reported recently in an fMRI study that revealed that LA individuals showed a reduced amygdala response to unattended fearful faces, whereas HA participants had no such reduction but instead showed an increased amygdala response to fearful versus neutral faces regardless of attentional focus (Bishop, Duncan, \& Lawrence, 2004). Enhanced amygdala activity in anxious individuals is, however, inconsistent with the significant $\mathrm{AB}$ effect for fearful faces that we observed in the HA group (although it was attenuated in comparison with the AB for happy expressions). The source of this apparent inconsistency is not clear, but it may be the case that attentional modulation is somewhat stronger in the AB task that we used than in the attentional task used by Bishop, Duncan, and Lawrence. This is a question that would be worth pursuing in future research.

For HA people, AB effects were attenuated for fearrelevant stimuli (fearful faces) relative to positively valenced stimuli (happy faces) that were rated as being equally emotional and arousing. We would not want to conclude, however, that the arousing nature of the stimuli is not important for $\mathrm{AB}$ effects. First, there is direct empirical evidence that the arousing qualities of affective stimuli are crucially important (Keil \& Ihssen, 2004). Second, our ratings of arousal were rather simplistic and it is likely that a more detailed analysis may reveal that fearful expressions are indeed more inherently arousing than happy expressions. The main point of our study is the demonstration that individual variations within the normal range of trait 

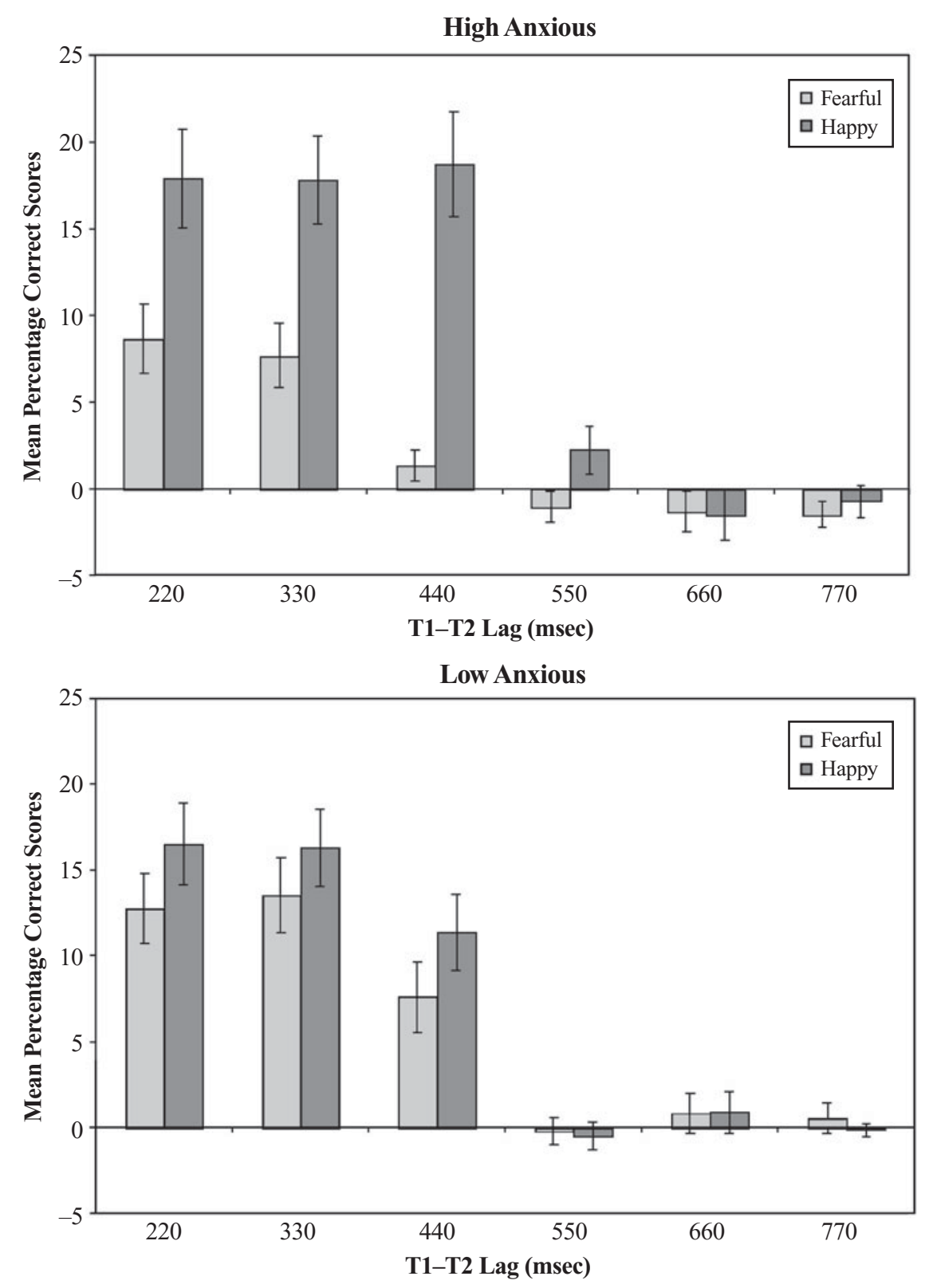

Figure 3. Mean attentional blink scores (mean correct for dual-task trials - mean correct for single-task trials) as a function of anxiety status (high vs. low), valence of T2 (fearful vs. happy), and SOA (220-770 msec).

and state anxiety can significantly modulate the degree of attention required for the processing of threat-relevant stimuli. Whereas attention gates the processing of a positive facial expression (happy face) for HA individuals, faces with a fearful expression do tend to break through the attentional blink for this group. This is consistent with models of anxiety-related bias that assume that anxiety represents a hypervigilance of the fear-detection system (see, e.g., Eysenck, 1992). The results are also consistent with models that suggest that anxiety is characterized by a reduced ability to exert top-down attentional control on the processing of threat-related stimuli (see, e.g., Derryberry \& Reed, 2002). Supporting evidence comes from a recent finding that the degree of amygdala activation in response to unattended fearful faces is indeed modulated by the level of self-reported anxiety (Bishop, Duncan, \& Lawrence, 2004). In the same group of participants, these researchers also found that the rostral ACC was not recruited as strongly in those with increased levels of state anxiety when they were presented with fearful facial expressions (Bishop, Duncan, Brett, \& Lawrence, 2004). The rostral ACC is considered to be an important com- 
ponent of the neural circuitry underlying top-down attentional control. The present results, together with those of Bishop, Duncan, Brett, and Lawrence (2004) and Bishop, Duncan, and Lawrence (2004), suggest that the pattern of attentional bias observed on behavioral tasks, as well as the pattern of neural activations in response to threatrelated stimuli, are strongly modulated by individual differences in the level of self-reported anxiety. Further integration of the clinical cognition literature with neuroscientific methods is likely to advance our understanding of the nature of cognitive processing biases in anxiety, leading to better theories and better treatment strategies for anxiety disorders. In addition, inclusion of measures of anxiety in neuroimaging studies is likely to improve our understanding of the complex interrelations between attention and emotion in the human brain.

\section{REFERENCES}

Amaral, D. G., \& Price, J. L. (1984). Amygdalo-cortical projections in the monkey (Macaca fascicularis). Journal of Comparative Neurology, 230, 465-496.

Anderson, A. K., \& Phelps, E. A. (2001). Lesions of the human amygdala impair enhanced perception of emotionally salient events. $\mathrm{Na}$ ture, 411, 305-309.

Arend, I., \& Botella, J. (2002). Emotional stimuli reduce the attentional blink in sub-clinical anxious subjects. Psicothema, 14, 209214.

Armony, J. L., \& Dolan, R. J. (2001). Modulation of spatial attention by masked angry faces: An event-related fMRI study [Abstract]. NeuroImage, 13, S378.

Barnard, P. J., Ramponi, C., Battye, G., \& Mackintosh, B. (2005). Anxiety and the deployment of visual attention over time. Visual Cognition, 12, 181-211.

Bishop, S. J., Duncan, J., Brett, M., \& Lawrence, A. D. (2004). Prefrontal cortical function and anxiety: Controlling attention to threatrelated stimuli. Nature Neuroscience, 7, 184-188.

Bishop, S. [J.], Duncan, J., \& Lawrence, A. D. (2004). State anxiety modulation of the amygdala response to unattended threat-related stimuli. Journal of Neuroscience, 24, 10364-10368.

Derryberry, D., \& REeD, M. A. (2002). Anxiety-related attentional biases and their regulation by attentional control. Journal of Abnormal Psychology, 111, 225-236.

Driver, J., \& TiPPER, S. P. (1989). On the nonselectivity of "selective" seeing: Contrasts between interference and priming in selective attention. Journal of Experimental Psychology: Human Perception \& Performance, 15, 304-314.

Eastwood, J. D., Smilek, D., \& Merikle, P. M. (2001). Differential attentional guidance by unattended faces expressing positive and negative emotion. Perception \& Psychophysics, 63, 1004-1013.

Ekman, P., \& Friesen, W. V. (1975). Pictures of facial affect. Palo Alto, CA: Consulting Psychologists Press.

Esteves, F., Parra, C., Dimberg, U., \& Öhman, A. (1994). Nonconscious associative learning: Pavlovian conditioning of skin conductance responses to masked fear-relevant facial stimuli. Psychophysiology, 31, 375-385.

EysEncK, M. W. (1992). Anxiety: The cognitive perspective. Hove, U.K.: Erlbaum.

Fox, E. (1994). Attentional bias in anxiety: A defective inhibition hypothesis. Cognition \& Emotion, 8, 165-195.

Fox, E. (2002). Processing emotional facial expressions: The role of anxiety and awareness. Cognitive, Affective, \& Behavioral Neuroscience, 2, 52-63.

Fox, E., Lester, V., Russo, R., Bowles, R. J., Pichler, A., \& Dutton, K. (2000). Facial expressions of emotion: Are angry faces detected more efficiently? Cognition \& Emotion, 14, 61-92.

Fox, E., Russo, R., Bowles, R. J., \& Dutton, K. (2001). Do threaten- ing stimuli draw or hold attention in subclinical anxiety? Journal of Experimental Psychology: General, 130, 681-700.

Georgiou, G. A., Bleakley, C., Hayward, J., Russo, R., Dutton, K., Eltiti, S., \& Fox, E. (2005). Focusing on fear: Attentional disengagement from emotional faces. Visual Cognition, 12, 145-158.

Holmes, A., Vuilleumier, P., \& Eimer, M. (2003). The processing of emotional facial expression is gated by spatial attention: Evidence from event-related brain potentials. Cognitive Brain Research, 16, 174-184.

KeIL, A., \& IHSSEN, N. (2004). Identification facilitation for emotionally arousing verbs during the attentional blink. Emotion, 4, 23-35.

LAVIE, N. (1995). Perceptual load as a necessary condition for selective attention. Journal of Experimental Psychology: Human Perception \& Performance, 21, 451-468.

LAVIE, N., \& Fox, E. (2000). The role of perceptual load in negative priming. Journal of Experimental Psychology: Human Perception \& Performance, 26, 1038-1052.

Lavie, N., \& Tsal, Y. (1994). Perceptual load as a major determinant of the locus of selection in visual attention. Perception \& Psychophysics, 56, 183-197.

Mathews, A. M., Fox, E., Yiend, J., \& Calder, A. (2003). The face of fear: Effects of eye gaze and emotion on visual attention. Visual Cognition, 10, 823-835.

MogG, K., \& Bradley, B. P. (1999). Orienting of attention to threatening facial expressions presented under conditions of restricted awareness. Cognition \& Emotion, 13, 713-740.

Morris, J. S., Friston, K. J., Büchel, C., Frith, C. D., Young, A. W., Calder, A. J., \& Dolan, R. J. (1998). A neuromodulatory role for the human amygdala in processing emotional facial expressions. Brain, 121, 47-57.

Morris, J. S., Frith, C. D., Perrett, D. I., Rowland, D., Young, A. W., Calder, A. J., \& Dolan, R. J. (1996). A differential neural response in the human amygdala to fearful and happy facial expressions. Nature, 383, 812-815.

Morris, J. S., Öhman, A., \& Dolan, R. J. (1999). A subcortical pathway to the right amygdala mediating "unseen" fear. Proceedings of the National Academy of Sciences, 96, 1680-1685.

Öhman, A., Flykt, A., \& Esteves, F. (2001). Emotion drives attention: Detecting the snake in the grass. Journal of Experimental Psychology: General, 130, 466-478.

Öhman, A., Lundqvist, D., \& Esteves, F. (2001). The face in the crowd revisited: A threat advantage with schematic stimuli. Journal of Personality \& Social Psychology, 80, 381-396.

Pessoa, L., Kastner, S., \& Ungerleider, L. G. (2002). Attentional control of the processing of neutral and emotional stimuli. Cognitive Brain Research, 15, 31-45.

Pessoa, L., McKenna, M., Gutierrez, E., \& Ungerleider, L. G. (2002). Neural processing of emotional faces requires attention. Proceedings of the National Academy of Sciences, 99, 11458-11463.

Phillips, M. L., Williams, L. M., Heining, M., Herba, C. M., RusSEll, T., ANDreW, C., ET AL. (2004). Differential neural responses to overt and covert presentations of facial expressions of fear and disgust. NeuroImage, 21, 1484-1496.

Raymond, J. E., Shapiro, K. L., \& Arnell, K. M. (1992). Temporary suppression of visual processing in an RSVP task: An attentional blink? Journal of Experimental Psychology: Human Perception \& Performance, 18, 451-468.

Shapiro, K. L., Arnell, K. M., \& Raymond, J. E. (1997). The attentional blink. Trends in Cognitive Sciences, 1, 291-296.

Shapiro, K. L., Caldwell, J., \& Sorensen, R. E. (1997). Personal names and the attentional blink: A visual "cocktail party" effect. Journal of Experimental Psychology: Human Perception \& Performance, 23, 504-514.

SPIELberger, C. D. (1983). Manual for the State-Trait Anxiety Inventory. Palo Alto, CA: Consulting Psychologists Press.

Vuilleumier, P., Armony, J. L., Driver, J., \& Dolan, R. J. (2001). Distinct effects of attention and emotion on face processing in the human brain: An event-related fMRI study. Neuron, 30, 829-841.

Vuilleumier, P., Richardson, M. P., Armony, J. L., Driver, J., \& Dolan, R. J. (2004). Distant influences of amygdala lesion on visual cortical activation during emotional face processing. Nature Neuroscience, 7, 1271-1278. 
Vuilleumier, P., \& Schwartz, S. (2001a). Beware and be aware: Capture of spatial attention by fear-related stimuli in neglect. NeuroReport, 12, 1119-1122.

Vuilleumier, P., \& Schwartz, S. (2001b). Emotional facial expressions capture attention. Neurology, 56, 153-158.

Whalen, P. J., Rauch, S. L., Etcoff, N. L., McInerney, S. C., Lee, M. B., \& Jenike, M. A. (1998). Masked presentations of emotional facial expressions modulate amygdala activity without explicit knowledge. Journal of Neuroscience, 18, 411-418.
Williams, J. M. G., Mathews, A., \& MacLeod, C. (1996). The emotional Stroop task and psychopathology. Psychological Bulletin, 120, 3-24.

Yiend, J., \& Mathews, A. M. (2001). Anxiety and attention to threatening pictures. Quarterly Journal of Experimental Psychology, 54A, 665-681.

(Manuscript received March 11, 2005; revision accepted for publication September 26, 2005.) 